\title{
A Self-Organising Network based on Lightweight Agents
}

\author{
John Debenham and Ante Prodan \\ Faculty of Information Technology, \\ University of Technology, Sydney, Australia \\ \{debenham, aprodan\}@it.uts.edu.au
}

\begin{abstract}
A lightweight multiagent system is deployed at each node in a communications network with the aim of self-organising the network as usage alters. The distributed, light-weight, co-operative multiagent system guarantees scalability of the approach. As the solution is distributed it is unsuitable to achieve any global optimisation goal - it simply seeks to continually improve network performance as demands change. Algorithms are described for adjusting the communication channels and for adjusting the network links. Experiments show that the method is robust and delivers good performance.
\end{abstract}

\section{Introduction}

The work discussed is based on previous work in the area of mesh networking and in particular in distributed algorithms at Columbia University, Microsoft Research, University of Maryland and Georgia Institute of Technology. In particular: [1], [2], [3] and [4].

There are three principal inputs to this work that we assume are available to the proposed methods:

- A load model. Given any contiguous set of nodes in a mesh, the load model specifies the actual or desired level of traffic flowing into, or out of, nodes in that set.

- A load balancing algorithm. Given any contiguous set of nodes in a mesh and the load model for that set, the load balancing algorithm determines how the traffic is allocated to links in the mesh so as to reach its desired destination where it leaves the mesh.

- An interference model. Given any contiguous set of nodes in a mesh, the interference model stipulates the interference level that each node in the mesh gives to the other nodes in the mesh given a known level of background interference due to transmission devices that are external to the mesh.

The work described below makes no restrictions on these three inputs other than that they are available to every node in the mesh. The load model, and so too the load balancing algorithm, will only be of value to a method for self-organisation if together they enable future load to be predicted with some certainty. We assume that the load is predictable. 
Below we introduce some terms, concepts and notation. Section 2 describes the role of the load balancing algorithm that our methods take as a given input. The measurement of interference cost is discussed in Section 3. Methods for the adjusting the channels in a multi-radio mesh networks for predictable load are described in Section 4, and for adjusting the links in Section 5. Future plans are described in Section 6.

The discrete time intervals mentioned below, e.g. $t, t+1$, are sufficiently spaced to permit what has to be done to be done.

A node is a set of radio interfaces (or "antennae") where each interface is associated with a particular channel, together with a controller that (intelligently we hope) assigns the channel on each interface. Interfaces that are part of the same node are assumed to be 'close' topologically, but this is not important. We assume for simplicity that each interface has its own, independent MAC layer.

A link is a pair of interfaces where each interface is assigned the same channel. The idea is that two interfaces communicate through a shared link. That is, if an interface is part of a link its state will be "listening and transmitting", otherwise its state will be "listening only".

Notation: nodes are denoted by Latin letters: $a, b, c, \ldots$, the interfaces for node $a$ are denoted by: $a[i]$ for $i=1, \ldots$, and links are denoted by Greek letters: $\alpha, \beta, \gamma, \ldots$ The interfaces communicate using an illocutionary communication language that is defined informally (for the time being) with illocutions being encapsulated in quotation marks: “.".

For any node $n, S_{n}$ is the set of nodes in node $n$ 's interference range. Likewise, for any link $\alpha, S_{\alpha}$ is the set of links that contain nodes $n$ 's interference range $\forall n \in \alpha$.

Given a node $a$, define $V_{a}=\cup_{n \in S_{a}} S_{n}$.

$\Gamma_{x}^{t}$ is channel used by $x$ to communicate at time $t$ where $x$ may be either an interface or a link.

$f(\cdot, \cdot)$ is an interference cost function that is defined between two interfaces or two links. It estimates the cost of interference to one interface caused by transmission from the other interface. This function relies on estimates of the interference level and the level of load (i.e.: traffic volume). So this function requires an interference model and a load model. This function is described in Section 3.

An interface is either 'locked' or 'unlocked'. A locked interface is either locked because it has committed to lock itself for a period of time on request from another interface, or it is 'self-locked' because it has recently instigated one of the self-organisation procedures in Section 4.

The abbreviation SNIR means "signal to noise plus interference ratio".

\section{The Load Balancing Algorithm}

We assume that if the external demands on a set of nodes $S$ are known and that there is a load balancing algorithm - that may or may not be intelligent - that determines how the load is routed through $S$. Figure 1 shows a set of twelve nodes connected by a mesh that is shown as dashed lines. The load on the mesh is shown by the four solid arrows. We assume that the load balancing algorithm will determine how the load is allocated to the links in the mesh. 
Fig. 1. The load balancing algorithm determines the allocation of load.

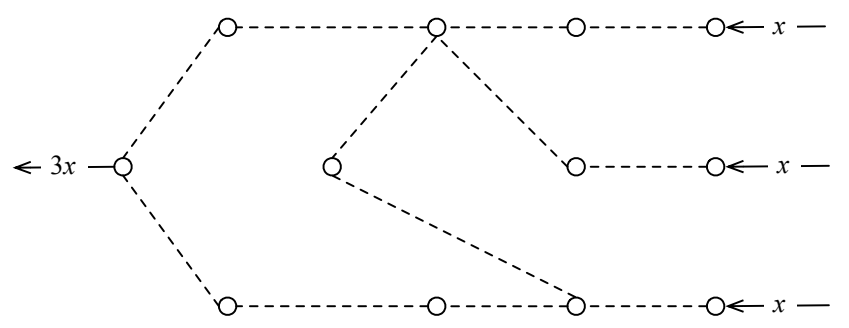

\section{Measuring Interference Cost}

Suppose that during some time interval $\Delta t$ two interfaces $a$ and $b$ are transmitting and receiving on channels $\Gamma_{a}$ and $\Gamma_{b}$. During $\Delta t$, the interference limit that interface $x$ imposes on interface $y, \tau_{y \mid x}$, is a ratio being the loss of traffic volume that interface $y$ could receive if interface $x$ were to transmit persistently divided by the volume of traffic that interface $y$ could receive if interface $x$ was silent:

$$
\tau_{y \mid x}=\frac{\left(m_{y} \mid \text { interface } x \text { silent }\right)-\left(m_{y} \mid \text { interface } x \text { persistent }\right)}{m_{y} \mid \text { interface } x \text { silent }}
$$

where $m_{y}$ is the mean SNIR observed by interface $y$ whilst listening on channel $\Gamma_{y}$, where as many measurements are made as is expedient in the calculation of this mean ${ }^{1}$. The interference load of each interface, $v_{a}$ and $v_{b}$, is measured as a proportion, or percentage, of some time interval during which that interface is transmitting. Then the observed interference caused by interface $b$ transmitting on channel $\Gamma_{b}$ as experienced by interface $a$ listening on channel $\Gamma_{a}$ is: $\tau_{a \mid b} \times v_{b}$, and the observed interference cost to interface $a$ is $^{2}$ :

$$
f(a \mid b) \triangleq \tau_{a \mid b} \times v_{b} \times\left(1-v_{a}\right)
$$

and so to interface $b$ :

$$
f(b \mid a)=\tau_{b \mid a} \times v_{a} \times\left(1-v_{b}\right)
$$

Now consider the interference between one interface $a$ and two other interfaces $c$ and $d$. Following the argument above, the observed interference caused by interfaces $c$ and $d$ as experienced by interface $a$ is $^{3}: \tau_{a \mid c} \times v_{c}+\tau_{a \mid d} \times v_{d}-\tau_{a \mid\{c, d\}} \times v_{c} \times v_{d}$. The observed interference cost to interface $a$ is:

$$
f(a \mid\{c, d\})=\left(1-v_{a}\right) \times\left(\tau_{a \mid c} \times v_{c}+\tau_{a \mid d} \times v_{d}-\tau_{a \mid\{c, d\}} \times v_{c} \times v_{d}\right)
$$

\footnotetext{
${ }^{1}$ For $\tau_{y \mid x}$ to have the desired meaning, $m_{y}$ should be a measurement of link throughput. However, link throughput and SNIR are approximately proportional — see [5].

${ }^{2}$ We assume here that whether or not interfaces $a$ and $b$ are transmitting are independent random events [6]. Then the probability that $a$ is transmitting at any moment is $v_{a}$, and the probability that $b$ is transmitting and $a$ is listening at any moment is: $\left(1-v_{a}\right) \times v_{b}$.

${ }^{3}$ That is, the interference caused by either interface $c$ or interface $d$.
} 
Suppose that $v_{\beta}$ is the proportion of $\Delta t$ for which either interface $c$ or interface $d$ is transmitting. Then for some $\kappa_{\beta}, 0 \leq \kappa_{\beta} \leq 1: v_{c}=\kappa_{\beta} \times v_{\beta}$, and $v_{d}=\left(1-\kappa_{\beta}\right) \times v_{\beta}$. Thus:

$$
f(a \mid \beta)=\left(1-v_{a}\right) \times v_{\beta} \times\left(\tau_{a \mid c} \times \kappa_{\beta}+\tau_{a \mid d} \times\left(1-\kappa_{\beta}\right)\right)
$$

Now suppose that interfaces $a$ and $b$ are linked, and that $v_{\alpha}$ is the proportion of $\Delta t$ for which either interface $a$ or interface $b$ is transmitting. Then for some $\kappa_{\alpha}, 0 \leq \kappa_{\alpha} \leq 1$ : $v_{a}=\kappa_{\alpha} \times v_{\alpha}, v_{b}=\left(1-\kappa_{\alpha}\right) \times v_{\alpha}$. Then as $a$ will only receive interference when it is listening to $b$ transmitting:

$$
f(a \mid \beta)=v_{b} \times v_{\beta} \times\left(\tau_{a \mid c} \times \kappa_{\beta}+\tau_{a \mid d} \times\left(1-\kappa_{\beta}\right)\right)
$$

and so:

$$
\begin{aligned}
f(\alpha \mid \beta)= & \left(1-\kappa_{\alpha}\right) \times v_{\alpha} \times v_{\beta} \times\left(\tau_{a \mid c} \times \kappa_{\beta}+\tau_{a \mid d} \times\left(1-\kappa_{\beta}\right)\right) \\
& +\kappa_{\alpha} \times v_{\alpha} \times v_{\beta} \times\left(\tau_{b \mid c} \times \kappa_{\beta}+\tau_{b \mid d} \times\left(1-\kappa_{\beta}\right)\right)
\end{aligned}
$$

Note that $v_{\alpha}, v_{\beta}, \kappa_{\alpha}$ and $\kappa_{\beta}$ are provided by the load model, and the $\tau_{x \mid y}$ are provided by the interference model.

\section{Adjusting the channels}

Our solution is based on the distinction in multiagent systems between proactive and reactive reasoning. Proactive reasoning is concerned with planning to reach some goal. Reactive reasoning is concerned with dealing with unexpected changes in the agent's environment. So in the context of self-organising networks we distinguish between:

- a reactive logic that deals with problems as they occur. The aim of our reactive module is simply to restore communication to a workable level that may be substantially sub-optimal.

- a proactive logic that, when sections of the network are temporarily stable, attempts to adjust the settings on the network to improve performance.

The reactive logic provides an "immediate fix" to serious problems. The proactive logic, that involves deliberation and co-operation of nearby nodes, is a much slower process.

Informally the proactive logic uses the following procedure:

- Elect a node $a$ that will manage the process

- Choose a link $\alpha$ from $a$ to another node - precisely a trigger criterion (see below) permits node $a$ to attempt to improve the performance of one of its links $\alpha \ni a$ with a certain priority level.

- Measure the interference

- Change the channel setting if appropriate

The following is a development of the ideas in [1]. 


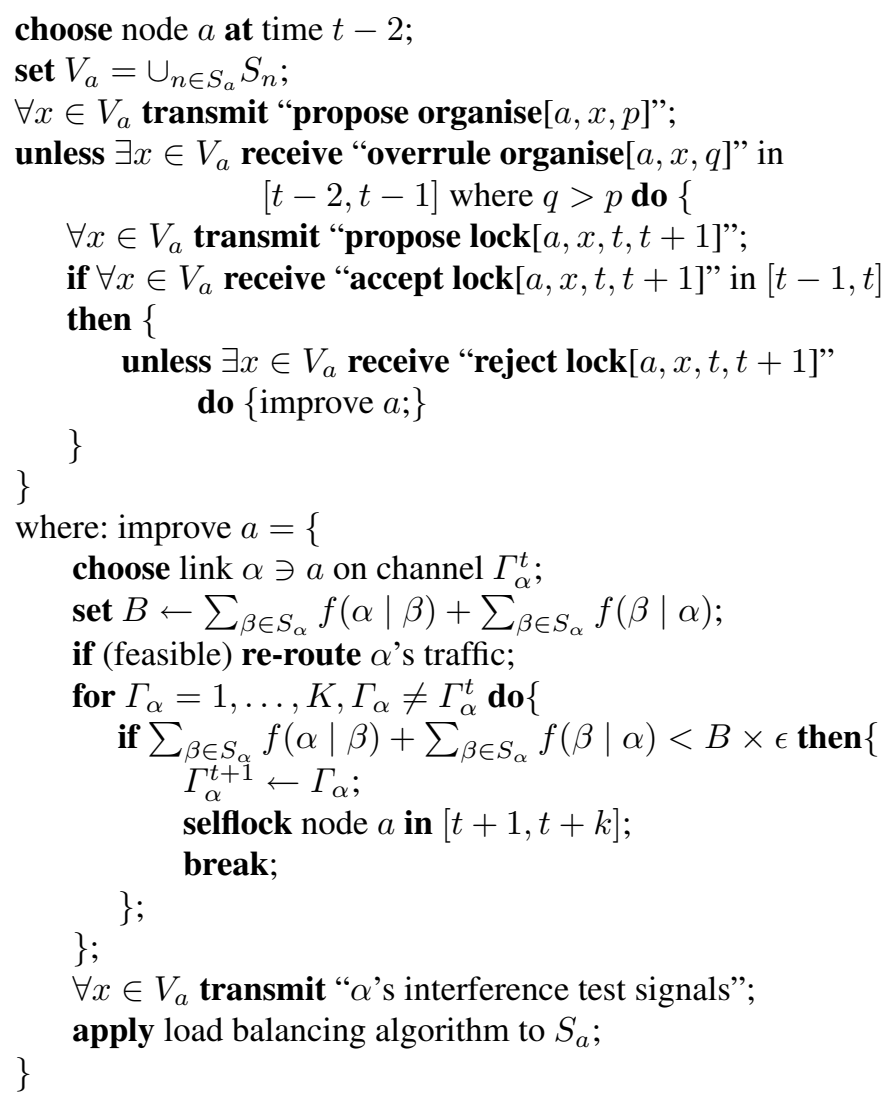

The statement selflock is to prevent $a$ from having to activate the method too frequently. The constant $\epsilon<1$ requires that the improvement be 'significant' both for node $a$ and for the set of nodes $S_{a}$. The stability of this procedure follows from the fact that it produces a net improvement of the interference cost within $S_{a}$. If a change of channel is effected then there will be no resulting change in interference outside $S_{a}$.

Interference model. We assume that each node, $a$, knows the channel of every node in $V_{a}$. We assume that each node is capable of measuring the strength of signals from every node in $V_{a}$. So if each node had access to all of this information from the point of view of every node in $V_{a}$, and, perhaps the level of background noise around $V_{a}$ then $a$ can derive estimates for the $\tau_{x \mid y}$ factors for all $x$ and $y$ in $V_{a}$. In particular, $a$ will be able to estimate all these factors to evaluate Equation 1 as required by the above algorithm. In addition, the procedure above suggests that if node $a$ is involved in changing its channel then at the end of this process - time permitting — it should transmit a 'beepsilence-beep-silence' message to enable every other node in $V_{a}$ to observe the actual $\tau$ values. Further, it is reasonable to suggest that this transmission of test signals could be carried out periodically in any case when network load permits. 
Fig. 2. Interference cost reduction as a function of node density.

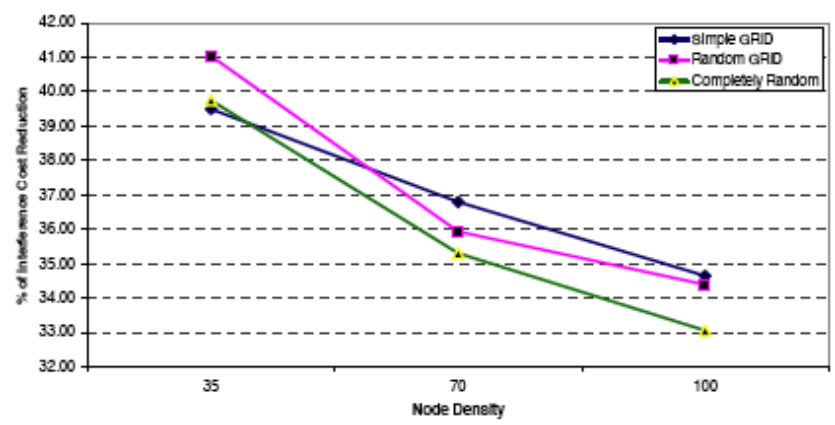

\subsection{Results and Discussion}

Impact of network (node) density on the performance. As the density of network increases (i.e. an increase in the number of routers located within the same area) the $I C$ reduction relatively decreases. This trend is shown across all the topologies. The impact of node density on the algorithm is relatively consistent for all topologies at the same router densities. From Figure 2 it can also be observed that the range of the interference reduction across the topologies at router densities of 35 routers and 100 routers is 1.55 and 1.58 , respectively.

Impact of typical topologies on the interference cost. Figure 3 shows the variation in the interference cost reduction as a function of network topology across different node densities. It can be deduced that the impact of the topologies on the performance of the algorithm (i.e. in terms of interference cost reduction) is insignificant. The mean of $I C$ reduction calculated from the data obtained shows that the topology with the smallest average $I C$ reduction is the completely random with a mean of 36.02 and topology with the most $I C$ reduction is the random grid with a mean of 37.12. The difference in performance between best and worst case is just 1.1 which confirms that the performance of the algorithm is almost completely independent of the type of topology.

Performance Comparison across the Network. In this study, we obtained interference cost $(I C)$ in different regions of the MR-WMN for the same set of links before and after the self-organisation algorithm is invoked. Comparison of the results obtained is shown in Figure 4 where the Interference cost is on the $X$-axis. From Figure 4 we can see that there were no nodes (red dots) that caused more interference after the self-organisation than it had caused before (blue dots) the self-organisation was invoked.

\section{Adjusting the links}

The algorithm for adjusting the links is precisely the same as the algorithm in Section 4 but with the following 'improve' methods. 
Fig. 3. Interference cost reduction as a function of topologies.

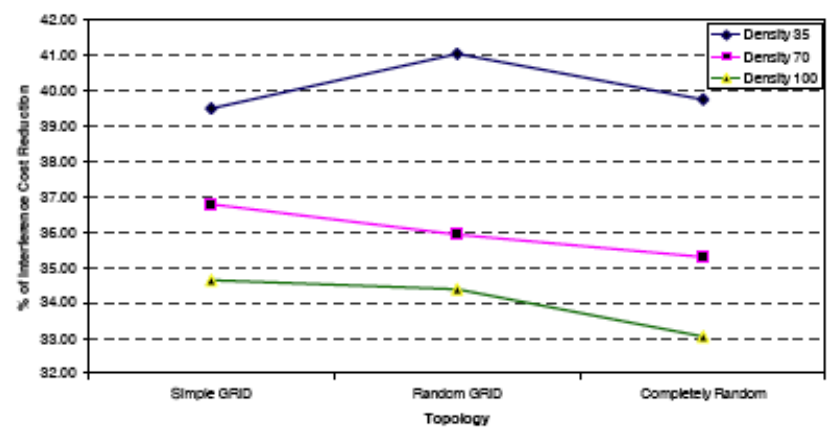

Fig. 4. Comparison of $I C$ across the network before (blue) and after (red) selforganisation.

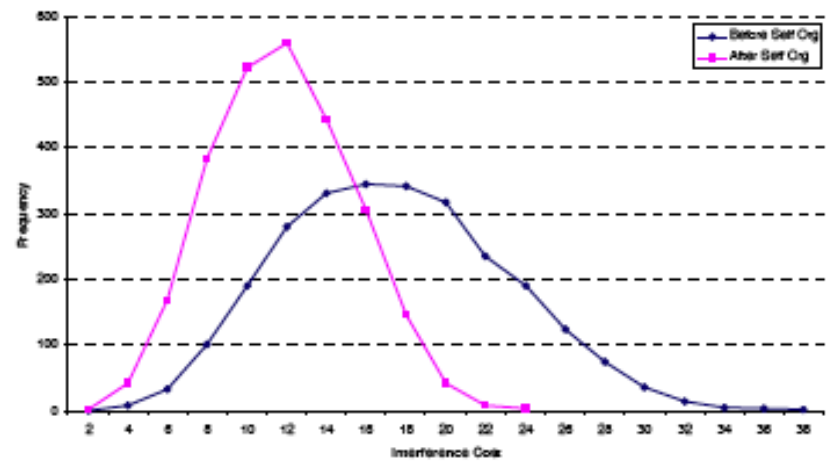

Link adjustment with known traffic load. Suppose that node $a$ has interference range $S_{a}$. Let $M_{a}$ be the set of nodes in $S_{a}$ excluding node $a$. Then use the method in Section 4 with the following 'improve' method:

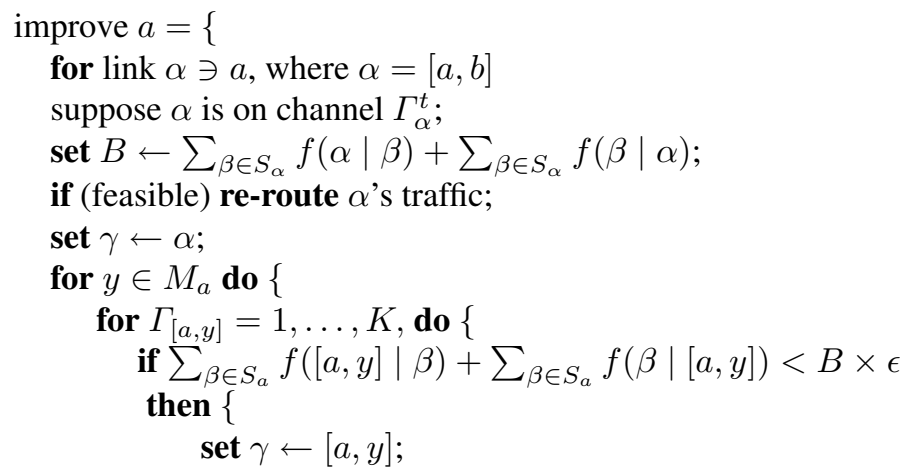




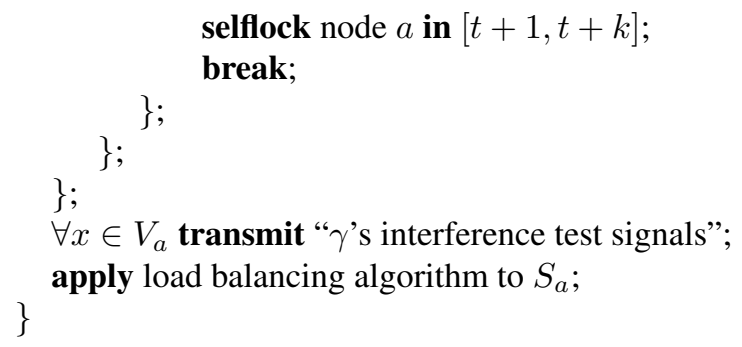

Trigger for attempting to adjust a link with known traffic load. Consider a mesh with known traffic load such as that illustrated in Figure 1. Suppose that the load balancing algorithm has allocated load to links on the mesh, and let link $(a, b)=\arg \max _{x \in N_{a}^{t}} \rho(x)$. If replacing $(a, b)$ with $(a, x)$ would mean that there exists a cut through the mesh that traverses $(a, x)$ and that all other links on that cut have a load $<\rho(a, b)$ then let node $a$ initiate the link adjusting procedure. Likewise if replacing $(a, b)$ with $(y, b)$. This is provisional. Have to double check. There could be a smarter way.

\subsection{Reactive Logic}

The relationship between the reactive and proactive logics is determined by:

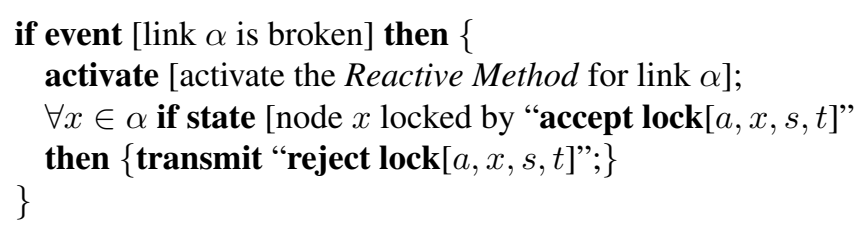

where the Reactive Method is as follows; it simply fixes disasters as they occur possibly with a configuration that is less satisfactory than the prior. It has no implications for neighbouring interfaces, and so it presents no instability issues.

Reactive Method. Important assumption for the functioning of the reactive logic discussed here is that all interfaces capable of reactive reconfiguration use omnidirectional antennas. The benefits and shortcomings of the usage of different antennas are discussed in details in our previous report. Two interfaces connected through directional antenna behave similarly to a wired point to point link because they cannot connect to any other interface to which their antennas are not aligned. This does not represent an impediment for the proposed architecture since majority of nodes will be equipped with omnidirectional antenna.

For the implementation of reactive logic we propose usage of simple mechanisms that are derived from routing protocols recently developed for stationary multi-radio mesh networks [4]. In conjunction with an appropriate routing protocol these mechanisms should ensure high reactivity in minimising effect of link interruptions caused by various factors. 
Fig. 5. Frequency distribution of the path length (in hops) without and with link substitution algorithm at 100 node network density and 10 additional links.

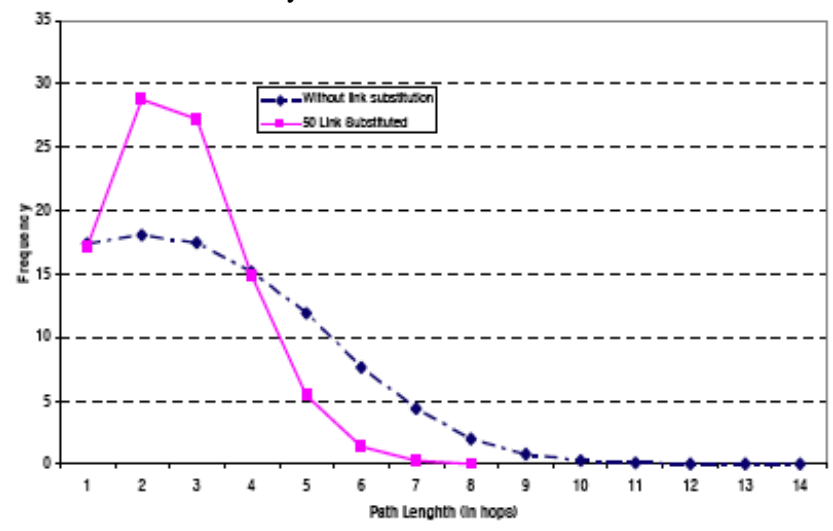

Link adjustment with unknown traffic load. Suppose that node $a$ has interference range $S_{a}$. Let $M_{a}$ be the set of nodes in $S_{a}$ excluding node $a$. For nodes $x, y \in S_{a}$, let $c(x, y)$ denote the cost ${ }^{4}$ of the least cost path that connects $x$ and $y$. We assume that: $(\forall x, y) c(x, y)=c(y, x)$, and that if the least cost path between nodes $u$ and $v$ is a subset of the least cost path between $x$ and $y$ then $c(u, v) \leq c(x, y)$. Let $N_{a}^{t}$ be the set of links in $S_{a}$ at time $t$, and $N_{a}^{t}(\ominus[a, x], \oplus[a, y])$ denotes the network configuration with link $[a, x]$ replaced by $[a, y]$. Let $C\left(N_{a}^{t}\right)$ denote the cost of the path of greatest cost in $S_{a}: C\left(N_{a}^{t}\right) \triangleq \max _{x, y \in S_{a}} c(x, y)$. Choose the pair of nodes $b$ and $c$ by:

$$
(b, c)=\arg \min _{(x, y) \mid[a, x] \in N_{a}^{t}, y \in M_{a}} C\left(N_{a}^{t}(\ominus[a, x], \oplus[a, y])\right)
$$

and swap link $[a, b]$ for link $[a, c]$ if:

$$
C\left(N_{a}^{t}(\ominus[a, b], \oplus[a, c])\right)<C\left(N_{a}^{t}\right) \times \epsilon
$$

where $\epsilon<1$ is a threshold constant [7].

\subsection{Results and Discussion}

This part of study firstly proposes the method for the link substitution that results with the reduction of the path length. Secondly, to provide the insight in algorithms effectiveness we produce over 3000 simulations. The simulation results are statistically processed and the outcomes for 3 different densities (35,70 and 100) are obtained.

Simulation parameters. We have used a Java based framework to carry out the simulations for the results shown and discussed in this section. The key attributes of the simulation were:

\footnotetext{
${ }^{4}$ The precise meaning of this cost function does not matter. It could be simply the number of hops, or some more complex measure involving load and/or interference.
} 
- Number of interfaces per router was randomly selected from 3 to 5 .

- Default signal strength was $100 \mathrm{~mW}(20 \mathrm{dBm}$ - Signal strength for each interface was randomly generated with $+/-25 \%$ variation.

- Network size had an area of $750 \mathrm{~m} \times 500 \mathrm{~m}$

In addition to the simulation parameters described above we limited the number of links to $n-1$; where $n$ is number of router (density) in a network. Consequently, the number of links created was 34,69 and 99 for the corresponding network densities. In addition to these link numbers we tested the effectiveness of the link substitution algorithm by creating additional 10 links when link substitution reached efficiency threshold. The number of the substituted link was limited in all simulation to $(10,20,30,40$ and 50) and separate results are shown. We now compare path lengths with and without link substitution. From the Figure 5 we can observer that our method significantly reduces path length by eliminating longer paths (maximum path length is 8 with the link substitution and 14 without it). This method also increases the number of shortest path (in particular paths 2 and 3 hops long).

\section{Conclusion and Future Work}

Through the work described in this report we have examined motivation and developed an algorithm for the topological control of MR-WMN. The goal of this algorithm is to increase the number of shortest paths to the portal nodes without adversely effecting interference cost. In addition to interference cost reduction implementation of this algorithm on MR-WMN further improve the system capacity.

Our future work will be focused on the development of our Java framework that is multi threaded so each node is represented as an independent thread. We believe that this will enable us to develop algorithms for tuning the capacity of the network links according to fluctuations in demand by mobile users.

\section{References}

1. Ko, B.J., Misra, V., Padhye, J., Rubenstein, D.: Distributed Channel Assignment in MultiRadio 802.11 Mesh Networks. Technical report, Columbia University (2006)

2. Mishra, A., Rozner, E., Banerjee, S., Arbaugh, W.: Exploiting partially overlapping channels in wireless networks: Turning a peril into an advantage. In: ACM/USENIX Internet Measurement Conference. (2005)

3. Mishra, A., Shrivastava, V., Banerjee, S.: Partially Overlapped Channels Not Considered Harmful. In: SIGMetrics/Performance. (2006)

4. Akyildiz, I.F., Wang, X., Wang, W.: Wireless mesh netowrks: a survey. Computer Networks (2005) 445-487

5. Vasudevan, S.: A Simulator for analyzing the throughput of IEEE 802.11b Wireless LAN Systems. Master's thesis, Virginia Polytechnic Institute and State University (2005)

6. Leith, D., Clifford, P.: A self-managed distributed channel selection algorithm for wlans. In: Proceedings of RAWNET, Boston, MA, USA (2006) 1-9

7. Ramachandran, K., Belding, E., Almeroth, K., Buddhikot, M.: Interference-aware channel assignment in multi-radio wireless mesh networks. In: Proceedings of Infocom 2006, Barcelona, Spain (2006) 1-12 